\title{
Observations of gamma-ray sources with DAMPE
}

\section{Kai-Kai Duan, ${ }^{a, *}$ Wei Jiang, ${ }^{a}$ Xiang Li ${ }^{a}$ Zhao-Qiang Shen ${ }^{a}$ and Zun-Lei $\mathbf{X u}^{a}$ on behalf of the DAMPE Collaboration \\ (a complete list of authors can be found at the end of the proceedings)}

${ }^{a}$ Key Laborary of Dark Matter and Space Astronomy, Purple Mountain Observatory, Yuan Hua Road, Nanjing, China

E-mail: duankk@pmo.ac.cn

DArk Matter Particle Explorer (DAMPE), a space-borne high energy cosmic ray and gamma-ray detector, has surveyed the whole sky for five years and collected more than 220,000 gamma-ray events above $2 \mathrm{GeV}$ since the launching on Dec. 17, 2015. The instrument response functions (IRFs) are derived and a dedicated software named DmpST is developed for the gamma-ray data analysis of DAMPE. Here we present the method of DAMPE bright gamma-ray sources search and the spectral analyses of these sources.

$37^{\text {th }}$ International Cosmic Ray Conference (ICRC 2021)

July 12 th - 23rd, 2021

Online - Berlin, Germany

\footnotetext{
*Presenter
} 


\section{Introduction}

The DArk Matter Particle Explorer (DAMPE) is a space-borne high energy particle detector dedicated to measure charged cosmic rays and gamma-rays in a broad energy range $[1,2]$ for the study of high energy astrophysics as well as the nature of dark matter particles[3, 4]. It consists of a Plastic Scintillator strip Detector (PSD), a Silicon-Tungsten tracKer-converter (STK), a BGO imaging calorimeter (BGO) and a NeUtron Detector (NUD)(Fig 1).

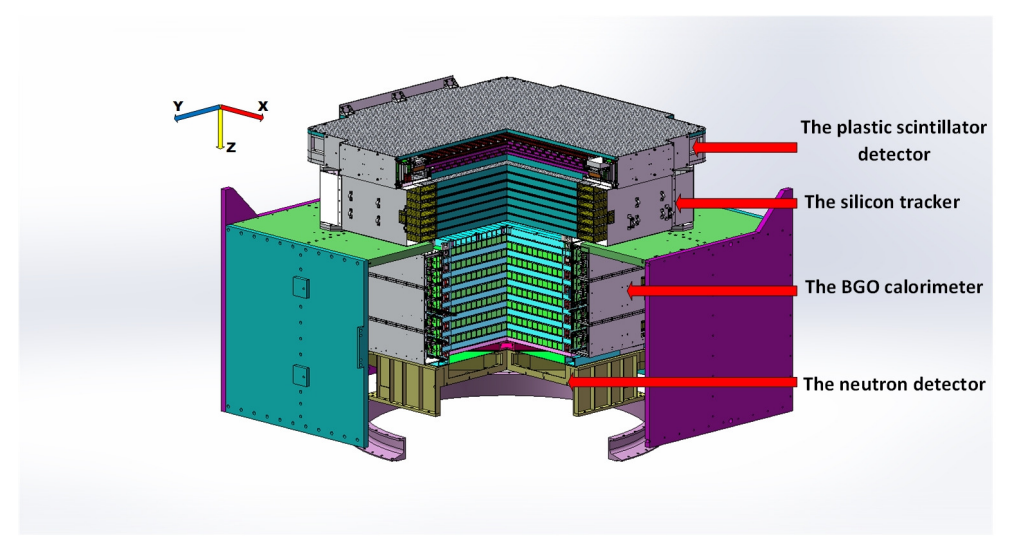

Figure 1: Schematic view of the DAMPE detector.

For gamma-ray observation, the energy range of DAMPE is from $2 \mathrm{GeV}$ to $10 \mathrm{TeV}$, the effective area is about $1200 \mathrm{~cm}^{2}$ for normal incident events, the angular resolution is about $0.2^{\circ}$ and the energy resolution is about $1 \%$ at $100 \mathrm{GeV}$ [2]. DAMPE is operating in a $500 \mathrm{~km}$ solar-synchronous orbit with an inclination of $\sim 97^{\circ}$ and a period of about 95 minutes. During the on-orbit operation, we have been carrying out its calibrations since its launch[5]. All these calibrations ensured the accuracy of the data.

\section{Gamma-ray data of DAMPE}

After more than five years' operation, DAMPE have collected more than 220,000 gamma-ray events above $2 \mathrm{GeV}$, selected from all events detected by DAMPE with gamma-ray photon selection algorithm [6]. We have carried out the calibration of boresight alignment for gamma-ray data [7], and derived the instrument response functions and developed a dedicated software named DmpST for gamma-ray data analysis [8].

In this work, we select the first five years' gamma-ray data from 1 Jan. 2016 to 31 Dec. 2020 and remove the events when DAMPE travels through the South Atlantic Anomaly (SAA) and during the solar flare time. Fig 2 shows the counts map of DAMPE five years' gamma-ray data in galactic coordinate. Fig 3 shows the time and energy distribution of DAMPE five years gamma-ray data. The periodicity of time distribution is caused by the orbit of DAMPE which surveys the whole sky per year. 


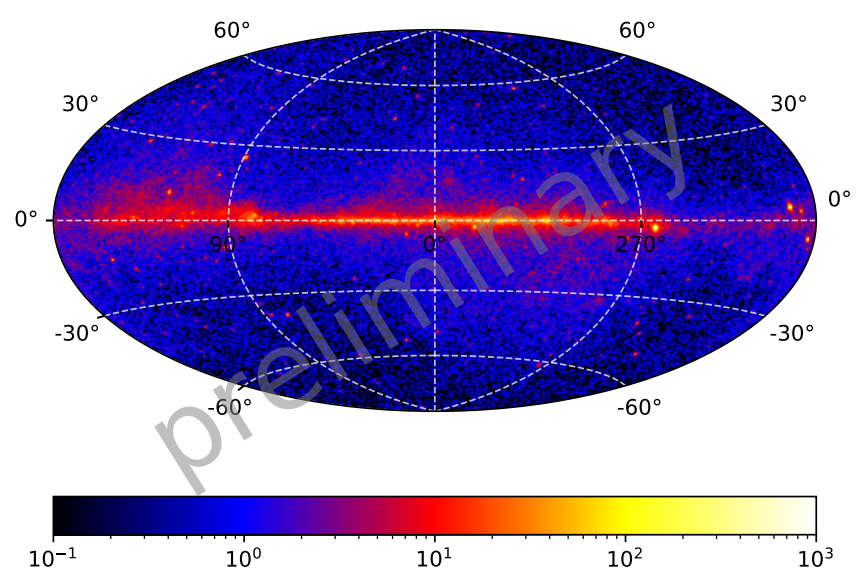

Figure 2: Counts map of DAMPE five years gamma-ray data in galactic coordinate with Aitoff projection.
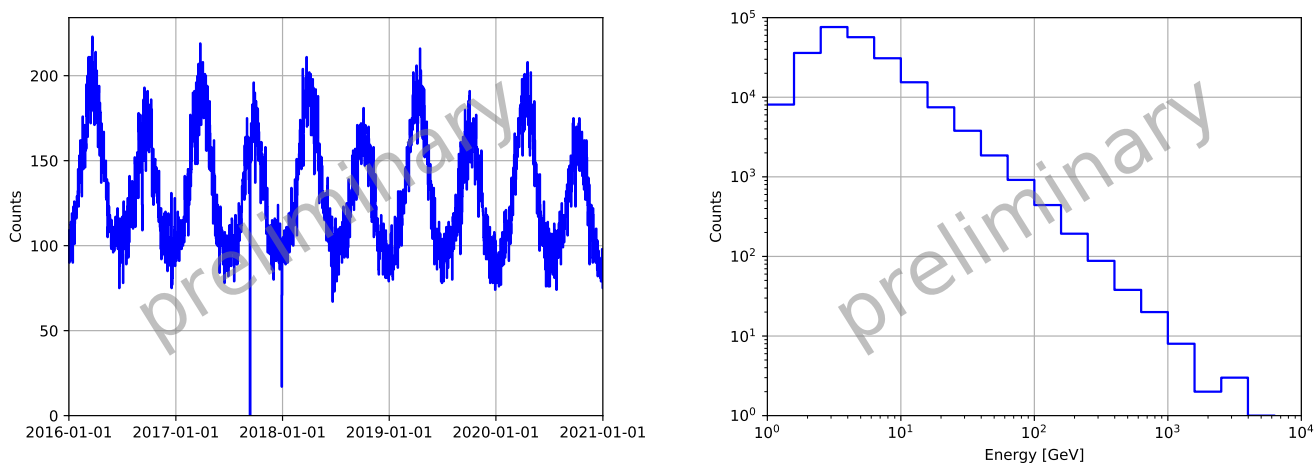

Figure 3: Time and energy distribution of DAMPE five years gamma-ray data.

\section{Method of blind search for source candidates}

The gamma-ray data contains three parts: galactic diffuse emission, resolved sources and isotropic diffuse emission. For detecting the resolved sources effectively, we apply the Li-Ma method[9] to blind search the source candidates firstly. We estimate the expected contribution of galactic diffuse emission with the model from Fermi-LAT's observations[10]. The significance of on region is defined as:

$$
S=\sqrt{2} \sqrt{N_{\text {on }}^{\prime} \ln \left[\frac{1+\alpha}{\alpha}\left(\frac{N_{\text {on }}^{\prime}}{N_{\text {on }}^{\prime}+N_{\text {off }}^{\prime}}\right)\right]+N_{\text {off }}^{\prime} \ln \left[(1+\alpha)\left(\frac{N_{\text {off }}^{\prime}}{N_{\text {on }}^{\prime}+N_{\text {off }}^{\prime}}\right)\right]},
$$

where $N_{\text {on }}^{\prime}=N_{\text {on }}-N_{\text {gal, on }}, N_{\text {off }}^{\prime}=N_{\text {off }}-N_{\text {gal,off }}, N_{\text {gal,on }}$ and $N_{\text {gal,off }}$ are the expected number of photons contributed by galactic diffuse emission in the on and off region. $\alpha$ is the ratio of exposure between off region and on region. We binned the data into more than 3 million equal solid angle's 
pixels with $\mathrm{N}_{\text {side }}=512$ in HEALPix prejection[11] and chose each pixel as well as its 8 neighbours as on region. The angular size of one pixel and on region are $0.1^{\circ}$ and $0.3^{\circ}$. In order to exclude the effect of sources and galactic diffuse emission, two step are used to determine the off region. We chose the neighbours with $\mathrm{N}_{\text {side }}=16$ for each pixel as off region firstly to get the initial significance of sky, and then chose the pixels which located in the high galactic latitude $\left(|b|>30^{\circ}\right)$ and significance less than 2.5 as the off region to get the finial significance map. Fig 4 shows the significance map of DAMPE gamma-ray data with the Li-Ma method.

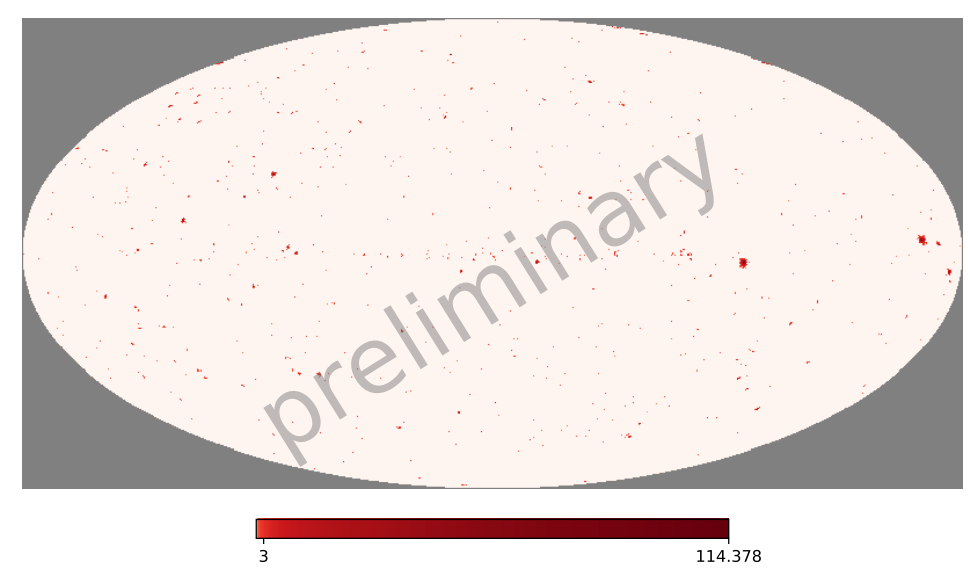

Figure 4: Significance map of DAMPE gamma-ray data with the Li-Ma method.

We chose the pixels which significance larger than 3 as the source candidates. Here we get 2130 candidates totally, and then we carried out likelihood analyses for each candidate with DmpST.

\section{Detection of gamma-ray sources}

We select the data around each candidate in 5 degree, and binned the data into 15 energy bin in log scale and 0.05 degree spatial bins. We set a point source with PowerLaw spectrum in each candidate's direction, and use the galactic diffuse model from Fermi-LAT's observation and isotropic diffuse model with PowerLaw spectrum to fit the data and get the TS value of source $T S_{\text {source }}=2 \ln \left(L_{\text {with }} / L_{\text {without }}\right)$. The 283 sources which TS value larger than 20 (TS > 20) make up the initial catalog. For reducing the effect of nearby sources, the sources in the 10 degree circle be included in the model to refit the data. The 222 sources which TS value larger than 25 (TS > 25) after the refit make up the finial catalog. Fig 5 shows the distribution of gamma-ray sources' flux and index observed by DAMPE fitted with the PowerLaw spectrum.

We also fit the data with curved spectrum including LogParabola $\frac{d N}{d E}=N_{0}\left(E / E_{b}\right)^{-\alpha+\beta \log \left(E / E_{b}\right)}$ and PLSuperExpCutoff $\frac{d N}{d E}=N_{0}\left(E / E_{0}\right)^{\gamma} \exp \left(-\left(E / E_{c}\right)^{b}\right)$ to get the TS value for curved spectrum $T S_{\text {curved }}=2 \ln \left(L_{\text {curved }} / L_{\text {Power Law }}\right)$. If the $T S_{\text {curved }}$ is larger than 9 , the significance for curved spectrum is larger than $3 \sigma$. We find that one source favored LogParabola spectrum and two sources favored PLSuperExpCutoff spectrum. Fig. 6 shows the SED of the sources with PowerLaw and curved spectra observed by DAMPE. 

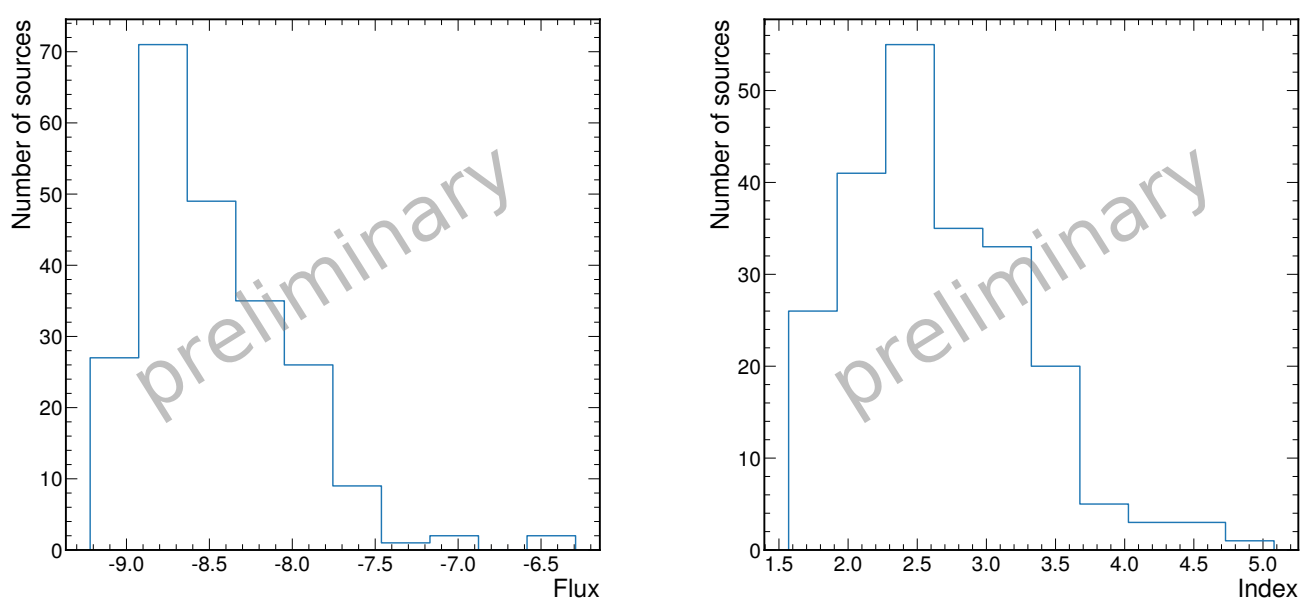

Figure 5: distribution of gamma-ray sources' flux and index observed by DAMPE fitted with the PowerLaw spectrum.

We associated these sources with Fermi-LAT's 4FGL[12] to detemine the types of these sources. We calculate the separations between the sources observed by DAMPE and sources in 4FGL, and consider the nearest source in 4FGL as the association of source observed by DAMPE. Fig 7 shows the separations distribution between sources observed by DAMPE and their associations in 4FGL. Fig 8 shows the types and spatial distribution of gamma-ray sources observed by DAMPE. Table 1 lists the types of sources observed by DAMPE.

Table 1: The types of sources observed by DAMPE.

\begin{tabular}{|c|c|c|c|c|c|c|}
\hline Type & AGN & Pulsar & SNR and/or PWN & binary & globular cluster & unassociated \\
\hline Number & 163 & 44 & 7 & 3 & 1 & 4 \\
\hline
\end{tabular}

\section{Summary}

After five years operation, DAMPE have collected more than 220,000 photons above $2 \mathrm{GeV}$. With the first five years' data, we detected 222 gamma-ray sources observed by DAMPE and detemine the spectra of these sources. Most of sources favors PowerLaw spectrum, and 3 sources favors curved spectra. Associated with 4FGL, we determined the types of sources. Most of sources are associated with AGNs and pulsars.

\section{Acknowledgments}

The DAMPE mission is funded by the strategic priority science and technology projects in space science of Chinese Academy of Sciences. In China the data analysis is supported in part by the National Key Research and Development Program of China (No. 2016YFA0400200), the National Natural Science Foundation of China (Nos. 11921003, U1738210, 12003074), the strategic 

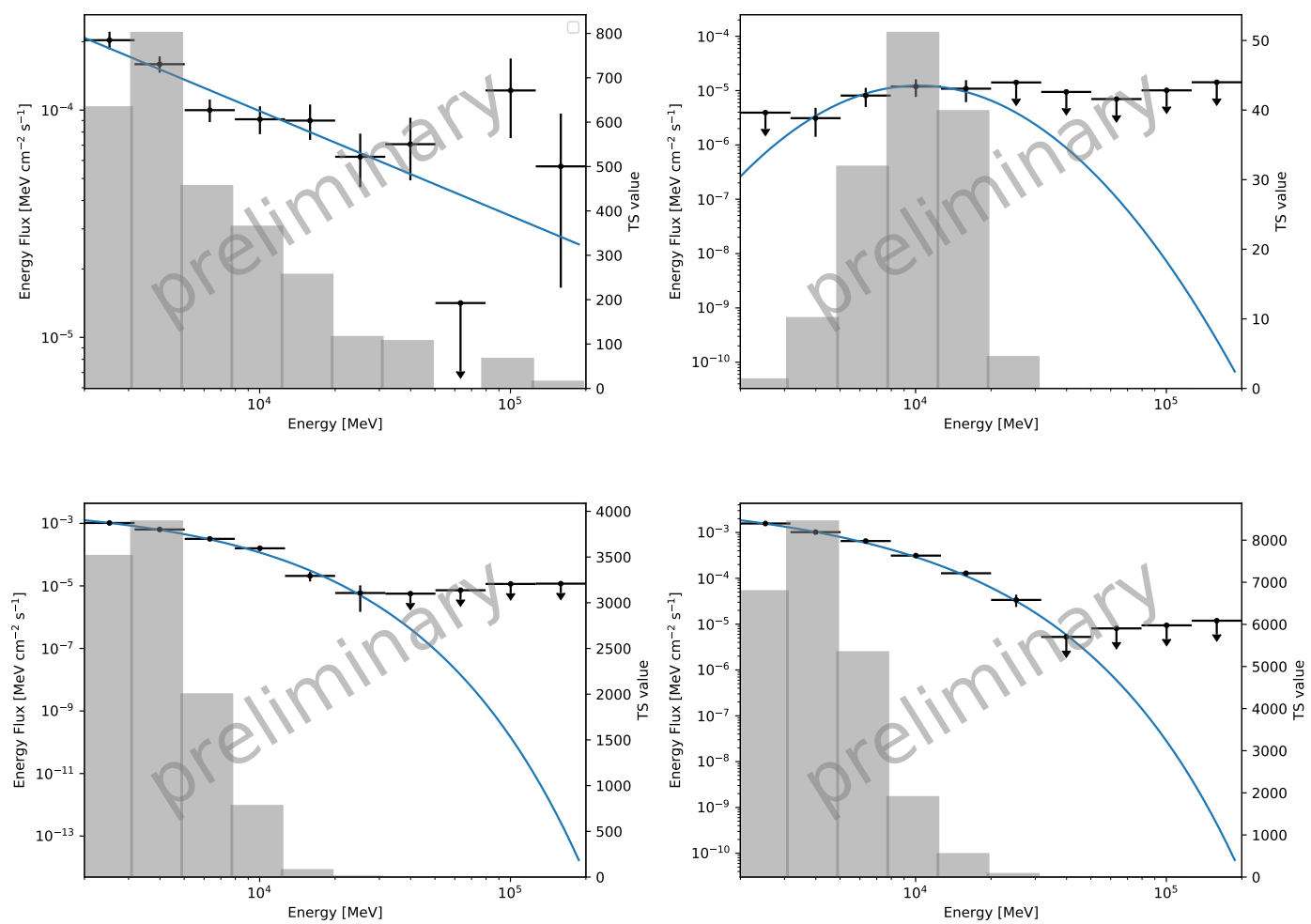

Figure 6: SED of sources with PowerLaw and curved spectra observed by DAMPE (Crab, 4FGL J1653.8+3945, Vela and Geminga).

priority science and technology projects of Chinese Academy of Sciences (No. XDA15051100), the Strategic Priority Research Program of Chinese Academy of Sciences (No. XDB23040401), the 100 Talents Program of Chinese Academy of Sciences, Youth Innovation Promotion Association CAS, and the Entrepreneurship and Innovation Program of Jiangsu Province. In Europe the activities and the data analysis are supported by the Swiss National Science Foundation (SNSF), Switzerland; the National Institute for Nuclear Physics (INFN), Italy.

\section{References}

[1] J. Chang, Dark Matter Particle Explorer: The First Chinese Cosmic Ray and Hard gamma-ray Detector in Space, Chin. J. Spac. Sci., 34 (2014) 550.

[2] J. Chang et al. (DAMPE Collaboration), The DArk Matter Particle Explorermission, Astropart. Phys., 95 (2017) 6.

[3] Y.-Z. Fan, B. Zhang and J. Chang, Electron/positron excesses in the cosmic ray spectrum and possible interpretations, Int. J. Mod. Phys. D, 19 (2010) 2011.

[4] J. L. Feng, Dark matter candidates from particle physics and methods of detection, Annu. Rev. Astron. Astrophys., 48 (2010) 495. 


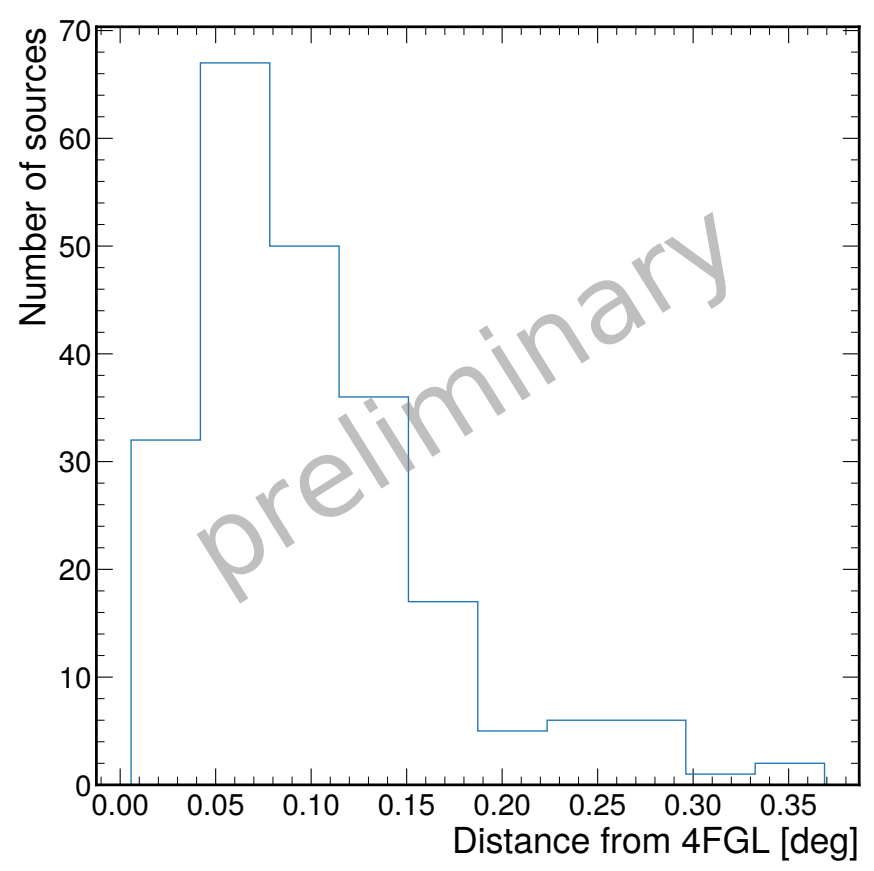

Figure 7: Separation distribution between sources observed by DAMPE and their associations in 4FGL.

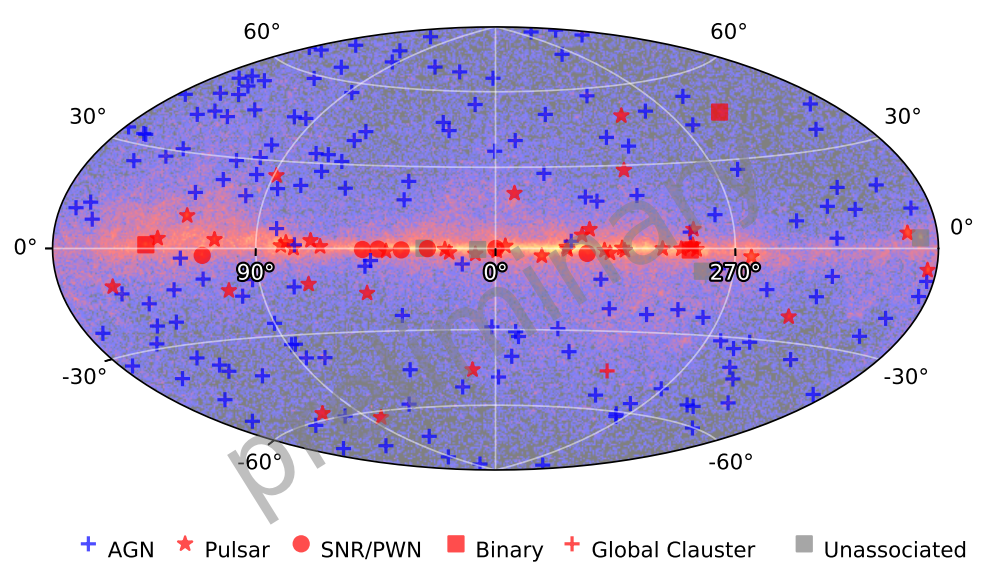

Figure 8: Types and spatial distribution of gamma-ray sources obseved by DAMPE

[5] G. Ambrosi et al. (DAMPE Collaboration), The on-orbit calibration of DArk Matter Particle Explorer, Astropart. Phys., 106 (2019) 18.

[6] Z.-L. Xu, K.-K. Duan, Z.-Q. Shen, et al., An algorithm to resolve gamma-rays from charged cosmic rays 162 with DAMPE, Research in Astronomy and Astrophysics, 18 (2018) 027. 
[7] W. Jiang, X. Li, K.-K. Duan, et al., The boresight alignment of the DArk Matter Particle Explorer, Research in Astronomy and Astrophysics, 20 (2020) 092.

[8] K.-K. Duan, W. Jiang, Y.-F. Liang, et al., DmpIRFs and DmpST: DAMPE instrument response functions and science tools for gamma-ray data analysis, Research in Astronomy and Astrophysics, 19 (2019) 132.

[9] T.-P. Li and Y.-Q. Ma, Analysis methods for results in gamma-ray astronomy, Astrophy. J, 272 (1983) 317.

[10] Acero F, Ackermann M, Ajello M, et al., Development of the Model of Galactic Interstellar Emission for Standard Point-source Analysis of Fermi Large Area Telescope Data, Astrophy. J. Supp., 223 (2016) 26.

[11] K. M. Górski, E. Hivon, A. J. Banday et al., HEALPix: A Framework for High-Resolution Discretization and Fast Analysis of Data Distributed on the Sphere, Astrophy. J., 622 (2005) 2.

[12] S. Abdollahi, F. Acero, M. Ackermann, et al., Fermi Lager Area Telescope Fourth Source Catalog, Astrophy. J. Supp., 247 (2020) 33. 


\section{Full Authors List: DAMPE Collaboration}

F. Alemanno ${ }^{1,2}$, Q. An ${ }^{3,4}$, P. Azzarello ${ }^{5}$, F. C. T. Barbato ${ }^{1,2}$, P. Bernardini ${ }^{6,7}$, X. J. Bi ${ }^{8,9}$, M. S. Cai ${ }^{10,11}$, E. Casilli6, ${ }^{6,7}$, E. Catanzani ${ }^{12}$, J. Chang ${ }^{10,11}$, D. Y. Chen ${ }^{9,10}$, J. L. Chen ${ }^{13}$, Z. F. Chen ${ }^{10,11}$, M. Y. Cui ${ }^{10}$, T. S. Cui ${ }^{14}$, Y. X. Cui ${ }^{10,11}$, H. T. Dai ${ }^{3,4}$, A. De Benedittis ${ }^{6,7}$, I. De Mitri ${ }^{1,2}$, F. de Palma ${ }^{6,7}$, M. Deliyergiyev ${ }^{5}$, M. Di Santo ${ }^{6,7}$, Q. Ding ${ }^{10,11}$, T. K. Dong ${ }^{10}$, Z. X. Dong ${ }^{14}$, G. Donvito ${ }^{15}$, D. Droz ${ }^{5}$, J. L. Duan ${ }^{13}$, K. K. Duan ${ }^{10}$, D. D’Urso ${ }^{12,1}$, R. R. Fan ${ }^{8}$, Y. Z. Fan ${ }^{10,11}$, K. Fang ${ }^{8}$, F. Fang ${ }^{13}$, C. Q. Feng ${ }^{3,4}$, L. Feng ${ }^{10}$, P. Fusco ${ }^{15,16}$, M.

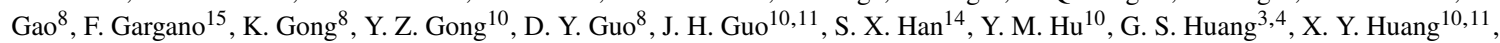

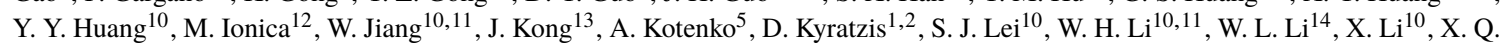

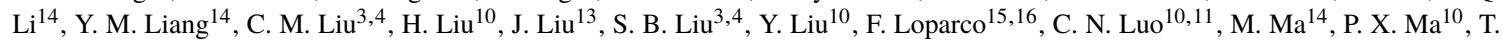
$\mathrm{Ma}^{10}$, X. Y. Ma ${ }^{14}$, G. Marsella ${ }^{6,7,2}$, M. N. Mazziotta ${ }^{15}$, D. Mo ${ }^{13}$, X. Y. Niu ${ }^{13}$, X. Pan ${ }^{10,11}$, A. Parenti ${ }^{1,2}$, W. X. Peng ${ }^{8}$, X. Y. Peng ${ }^{10}$, C. Perrina ${ }^{5,3}$, R. Qiao ${ }^{8}$, J. N. Rao ${ }^{14}$, A. Ruina ${ }^{5}$, M. M. Salinas ${ }^{5}$, G. Z. Shang ${ }^{14}$, W. H. Shen ${ }^{14}$, Z. Q. Shen ${ }^{10}$, Z. T. Shen ${ }^{3,4}$, L. Silveri ${ }^{1,2}$, J. X. Song ${ }^{14}$, M. Stolpovskiy ${ }^{5}$, H. Su ${ }^{13}$, M. Su${ }^{17}$, H. R. Sun ${ }^{3,4}$, Z. Y. Sun ${ }^{13}$, A. Surdo ${ }^{7}$, X. J. Teng ${ }^{14}$, A. Tykhonov ${ }^{5}$, H. Wang ${ }^{14}$, J. Z. Wang ${ }^{8}$, L. G. Wang ${ }^{14}$, S. Wang ${ }^{10,11}$, S. X. Wang ${ }^{10,11}$, X. L. Wang ${ }^{3,4}$, Y. Wang ${ }^{3,4}$, Y. F. Wang ${ }^{3,4}$, Y. Z. Wang ${ }^{10}$, D. M. Wei ${ }^{10,11}$, J. J. Wei ${ }^{10}$, Y. F. $\mathrm{Wei}^{3,4}$, D. $\mathrm{Wu}^{8}$, J. Wu ${ }^{10,11}$, L. B. Wu ${ }^{1,2}$, S. S. Wu ${ }^{14}$, X. Wu ${ }^{5}$, Z. Q. Xia ${ }^{10}$, E. H. Xu ${ }^{3,4}$, H. T. Xu ${ }^{14}$, Z. H. Xu ${ }^{10,11}$, Z. L. Xu ${ }^{10}$, Z. Z. Xu ${ }^{3,4}$, G. F. Xue ${ }^{14}$, H. B. Yang ${ }^{13}$, P. Yang ${ }^{13}$, Y. Q. Yang ${ }^{13}$, H. J. Yao ${ }^{13}$, Y. H. Yu ${ }^{13}$, G. W. Yuan ${ }^{10,11}$, Q. Yuan ${ }^{10,11}$, C. Yue ${ }^{10}$, J. J. Zang ${ }^{10,4}$, F. Zhang ${ }^{8}$, S. X. Zhang ${ }^{13}$, W. Z. Zhang ${ }^{14}$, Y. Zhang ${ }^{10}$, Y. J. Zhang ${ }^{13}$, Y. L. Zhang ${ }^{3,4}$, Y. P. Zhang ${ }^{13}$, Y. Q. Zhang $^{10}$, Z. Zhang ${ }^{10}$, Z. Y. Zhang ${ }^{3,4}$, C. Zhao ${ }^{3,4}$, H. Y. Zhao ${ }^{13}$, X. F. Zhao ${ }^{14}$, C. Y. Zhou ${ }^{14}$, and Y. Zhu ${ }^{14}$

${ }^{1}$ Gran Sasso Science Institute (GSSI), Via Iacobucci 2, I-67100 L'Aquila, Italy

${ }^{2}$ Istituto Nazionale di Fisica Nucleare (INFN) -Laboratori Nazionali del Gran Sasso, I-67100 Assergi, L'Aquila, Italy

${ }^{3}$ State Key Laboratory of Particle Detection and Electronics, University of Science and Technology of China, Hefei 230026, China

${ }^{4}$ Department of Modern Physics, University of Science and Technology of China, Hefei 230026, China

${ }^{5}$ Department of Nuclear and Particle Physics, University of Geneva, CH-1211, Switzerland

${ }^{6}$ Dipartimento di Matematica e Fisica E. De Giorgi, Universita‘ del Salento, I-73100, Lecce, Italy

${ }^{7}$ Istituto Nazionale di Fisica Nucleare (INFN) - Sezione di Lecce, I-73100, Lecce, Italy

${ }^{8}$ Institute of High Energy Physics, Chinese Academy of Sciences, Yuquan Road 19B, Beijing 100049, China

${ }^{9}$ University of Chinese Academy of Sciences, Yuquan Road 19A, Beijing 100049, China

${ }^{10}$ Key Laboratory of Dark Matter and Space Astronomy, Purple Mountain Observatory, Chinese Academy of Sciences, Nanjing 210023 , China

${ }^{11}$ School of Astronomy and Space Science, University of Science and Technology of China, Hefei 230026, China

${ }^{12}$ Istituto Nazionale di Fisica Nucleare (INFN) - Sezione di Perugia, I-06123 Perugia, Italy

${ }^{13}$ Institute of Modern Physics, Chinese Academy of Sciences, Nanchang Road 509, Lanzhou 730000, China

${ }^{14}$ National Space Science Center, Chinese Academy of Sciences, Nanertiao 1, Zhongguancun, Haidian district, Beijing 100190, China

${ }^{15}$ Istituto Nazionale di Fisica Nucleare (INFN) - Sezione di Bari, I-70125, Bari, Italy

${ }^{16}$ Dipartimento di Fisica "M. Merlin" dell'Universita' e del Politecnico di Bari, I-70126, Bari, Italy

${ }^{17}$ Department of Physics and Laboratory for Space Research, the University of Hong Kong, Pok Fu Lam, Hong Kong SAR, China

${ }^{1}$ Now at Universita' di Sassari, Dipartimento di Chimica e Farmacia, I-07100, Sassari, Italy.

${ }^{2}$ Now at Universita“ degli Studi di Palermo, Dipartimento di Fisica e Chimica "E. Segre“", via delle Scienze ed. 17, I-90128 Palermo, Italy.

${ }^{3}$ Also at Institute of Physics, Ecole Polytechnique Federale de Lausanne (EPFL), CH-1015 Lausanne, Switzerland.

${ }^{4}$ Also at School of Physics and Electronic Engineering, Linyi University, Linyi 276000, China. 